\title{
Development of a VOCs analyzer for measuring pesticide emission by a low-cost electronic nose
}

\author{
Hang Zhu' ${ }^{1}$, Yubin Lan ${ }^{2,3,4^{*}}$, Ningye Ding ${ }^{5}$, Ling Wang ${ }^{6}$ \\ (1. School of Mechanical and Aerospace Engineering, Jilin University, Changchun, 130025, China; \\ 2. National Center for International Collaboration Research on Precision Agricultural Aviation Pesticide Spraying Technology, \\ Guangzhou, 510642, China; \\ 3. School of Agricultural Engineering and Food Science, Shandong University of Technology, Zibo, 255000, China; \\ 4. College of Biological and Agricultural Engineering, Texas A\&M University, College station, 77843, USA; \\ 5. College of Engineering, Northeast Agricultural University, Harbin, 150030, China; \\ 6. College of Engineering, China Agricultural University, Beijng, 100083, China)
}

\begin{abstract}
This work aims to develop an analyzer which detects volatile organic compounds (VOCs) from pesticides applied via aerial spray. A metal-oxide-semiconductor (MOS) gas sensor was chosen for use as an electronic nose (e-nose). For the fabrication of the VOCs analyzer, and the reliable hardware and software designs, an airflow intake system was used to speed up the precise pesticide concentration analysis in the electronic nose system. A control logic was used for quantitative detection of the VOCs mixtures. To evaluate the performance of the VOCs analyzer, two kinds of pesticide were tested. The VOCs analyzer successfully distinguished the concentrations of both pesticides efficiently with a low cost, which promises the potential for use as a universal VOCs analysis platform for the detection of pesticide emissions. Using this VOCs analyzer, the relative error was $5.2 \%$ over field testing. It can be used to detect the concentration of pesticides in cotton fields since it is practical, feasible and accurate.
\end{abstract}

Keywords: e-nose, gas sensor, VOCs analyzer, pesticide emission

DOI: $10.33440 /$ j.ijpaa.20210401.152

Citation: Zhu H, Lan Y B, Ding N Y, Wang L. Development of a VOCs analyzer for measuring pesticide emission by a low-cost electronic nose. Int J Precis Agric Aviat, 2021; 4(1): 1-5.

\section{Introduction}

Cotton is one of the most important crops globally and is widely planted around the world with high annual yields ${ }^{[1]}$. In the United States, cotton acreage is up to $1.4 * 108$ ha, but economic losses in the cotton industry due to the stink bug pest are several million dollars annually ${ }^{[2]}$. Therefore, cotton pest control is critical to improve cotton production. Chemical control is one of the most significant strategies for the prevention and control of cotton diseases and insect pests ${ }^{[3-5]}$. In recent years, the use of broad-spectrum insecticides has effectively controlled bugs, worms and weevils, and greatly increased cotton production ${ }^{[6]}$. However, excessive use of pesticides has resulted in a resurgence of other pests, as well as the development of pesticide resistance ${ }^{[7]}$, which makes future pest control more difficult. Meanwhile, excessive

Received date: 2021-02-25 Accepted date: 2021-03-28

Biographies: Hang Zhu, $\mathrm{PhD}$, Associate professor, Research interests: precision agriculture aviation and equipment, Telephone number: +86-1808-866-5997, Email: hangzhu@jlu.edu.cn; Ningye Ding, PhD, Research interests: precision agriculture aviation and equipment, Telephone number: +86-1390-433-6777, Email: ningye-d@163.com; Ling Wang, PhD, Research interests: precision agriculture aviation and equipment, Telephone number: +86-010-6273-6591, Email: wangling.0928@163.com.

*Corresponding author: Yubin Lan, PhD, Director and Chief Scientist of the National International Joint Research Center for Precision Agricultural Aviation Pesticide Spraying Technology, Professor and doctoral supervisor of South China Agricultural University, Adjunct Senior Researcher at USDA, Adjunct Senior Researcher at Texas A\&M University Professor and doctoral Supervisor. Research interests: precision agriculture aviation and equipment. Mailing Address: College of Engineering, Northeast Agricultural University. Telephone number: +86-1392-270-7507, Email: ylan@scau.edu.cn. use of pesticides has greatly impacted ecological balances, polluted the environment, and harmed human health ${ }^{[8-12]}$. Therefore, it is important to develop more sustainable methods for the use of pesticides in order to both improve cotton production and minimize the negative effects of chemical control.

For the purpose of controlling pests and minimizing ecological damage, it is necessary to effectively identifying cotton pests and determining the exact amount of pesticide sufficient ${ }^{[13]}$. Some scholars have found that different odors are produced depending on pest species and the degree of host infestation ${ }^{[14]}$. Thus, odor detection can be used to determine pest species and degree of infestation. This can allow an electronic nose with appropriate sensors to accurately identify an infestation and evaluate how extensive it is. The electronic nose could not only be used to recognize single or composite odors, but also to detect gas, vapor or a mixture.

Electronic noses require a relatively stable working environment, because its detection accuracy can be significantly reduced by variables such as field temperature, atmospheric humidity and wind speed ${ }^{[15-19]}$. Meanwhile, it is difficult to measure the concentration of pesticides based on the ratio of pesticide. The inaccuracy of pesticide concentration along with variable environmental conditions will result in an uneven distribution of pesticide in a region ${ }^{[20-22]}$.

To deal with the challenges of environmental variation and the need for extended use, a VOCs analyzer was developed which has low power consumption for extended use, high efficiency and the ability to respond rapidly to the variation of field conditions. The VOCs analyzer consists of a metal oxide gas sensor (TGS822), a measuring circuit, software, with real-time gas collection, onboard 
data storage and analysis. The VOCs analyzer is able to measure pesticide concentration, and can monitor pest odors in fields in extended time.

\section{Materials and methods}

\subsection{System composition}

The small size and lightweight VOCs analyzer (Figure 1) mainly consists of a gas sensor, a detecting circuit and data analysis software. It can be connected to a computer through a RS232 cable and can collect and analyze odor data.

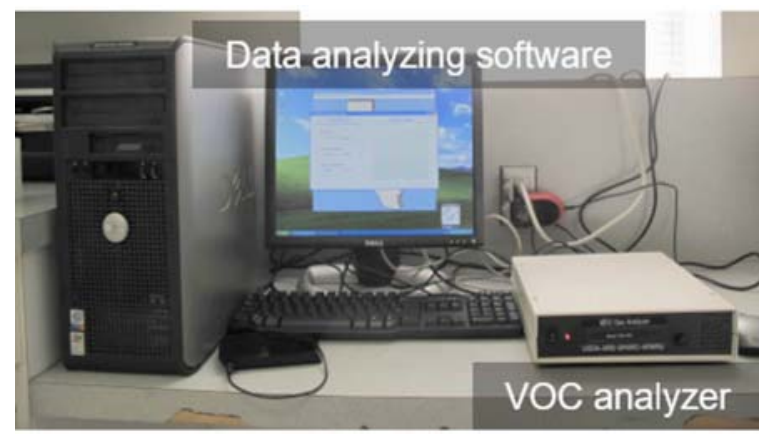

Figure 1 System composition of the VOCs analyzer

\subsubsection{Sensor selection}

A gas sensor is the core of VOCs analyzers, and its stability and sensitivity directly affect the performance of the system. This system is built with the commercially available metal oxide gas sensor, TGS822 (Figaro Engineering INC, Mino, Osaka, Japan). This sensor is used for gas detection, particularly for organic solvent vapors. It can work stably for an extended amount of time and has reliable performance, low power consumption and high efficiency.

The TGS822 sensor consists of a tin oxide semiconductor, which has low electrical conductivity in clean air. When the sensor detects targeted gas, the conductivity of the senor will change with the variation of gas concentration. Therefore, the system can use a circuit to regulate the output signal of the sensor when it contacts the gas. The system is used to detect pesticides, which consist of various compounds such as ethers and lipids that the TGS sensor is highly sensitive to.

The relation between the resistance of TGS822 sensors and gas concentration is in equation (1).

$$
R_{S}=A[C]^{-\alpha}
$$

Where, $R_{S}=$ resistance of sensor $(\mathrm{k} \Omega) ; A=$ constant; $C=$ gas concentration (ppm); $\alpha=$ slope of sensor resistance curve.

Resistance and associated parameters are listed on Table 1.

Table 1 The resistance parameter of sensor

\begin{tabular}{lcc}
\hline \multicolumn{1}{c}{ Title } & Symbol & Dimension \\
\hline Sensor resistance & $R_{S}$ & $1-10 \mathrm{k} \Omega$ \\
Rate of change in resistance & $R_{S} / R_{O}$ & $0.4 \pm 0.1$ \\
Heating resistor & $R_{H}$ & $38.0 \pm 3.0 \Omega$ \\
\hline
\end{tabular}

\subsubsection{Circuit design}

The detection circuit consists of a sensor measuring circuit, signal amplification, conditioning circuitry, and an analog-to-digital conversion circuit (Figure 2). This arrangement turns the detected variable quantity of gas to an output signal, and transmits through a RS232 cable to a computer which is then used for data processing.

The resistance value of the TGS sensor has a logarithmic relationship to the concentration of measured gas, and the sensitivity characteristic of the sensor is indicated by the ratio of the sensor resistance due to different gas concentration. The signal amplification and conditioning circuitry were needed to transmit the change of resistance value into a voltage variation. Through the circuitry, the voltage variation is amplified and transmit to an analog-to-digital conversion. The high-precision amplifier INA118 (Instrument Amplifier) was chosen for construction of a signal amplification and conditioning circuit, and the LTC1298 serial ADC conversion was used.

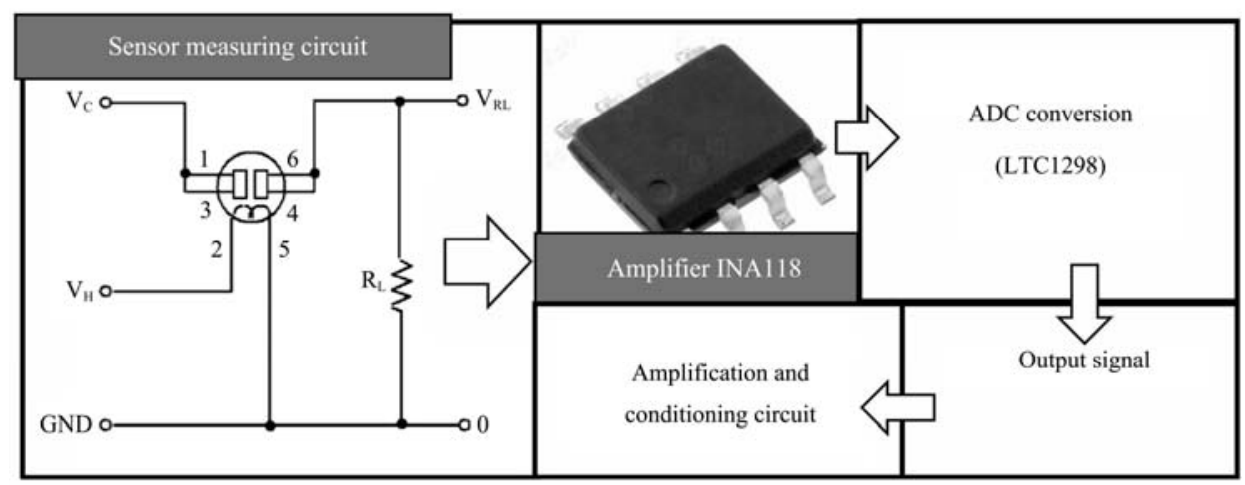

Figure 2 Measuring circuit

\subsubsection{Gas-route circuit design}

Gas-route design is the key to sampling detection, and data acquisition is accomplished in this section of the device. Through the contact between the gas and sensor, it will generate an electrical signal. This section consists of a fan and sensor (Figure 3), a requisite volume of gas enters into the collection chamber through the aperture of the VOCs analyzer and generate a suitable response from the sensor. Gas is drawn into the duct by the gas chamber fan, which facilitates sufficient gas contacting with the sensor, and the gas is then discharged from the vent on the back of the VOCs analyzer. Alternatively, the measured gas can be separated from the measurement circuit in the sampling channels to avoid circuit corrosion, so the reliability of the system can be improved during detection.

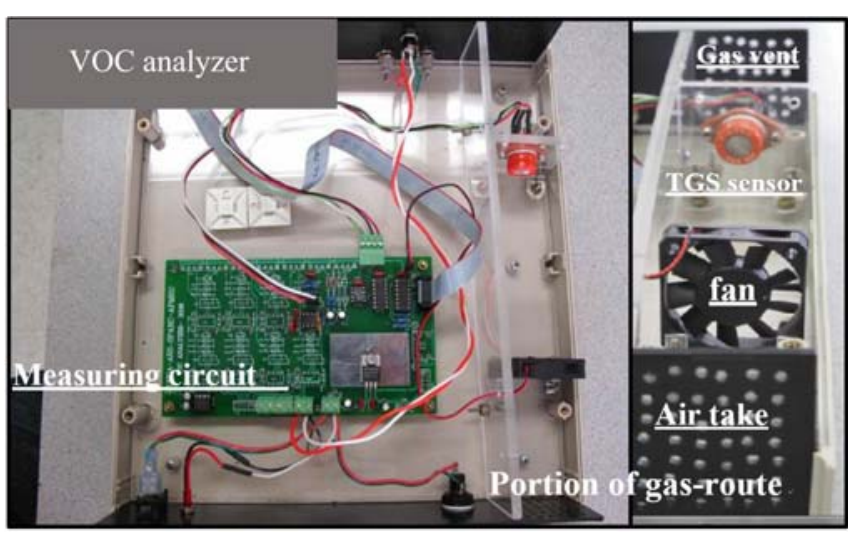

Figure 3 Gas sampling portion of VOC analyzer 


\subsection{Data analyzing and processing}

Due to the sensitivity of the sensor is different from various gases and air content varies between testing environments, it is necessary to calibrate the VOCs analyzer before testing. After calibration, it is necessary to adjust the instrument by using known gas concentrations. A flowchart for the data processing of the VOCs analyzer is shown in Figure 4.

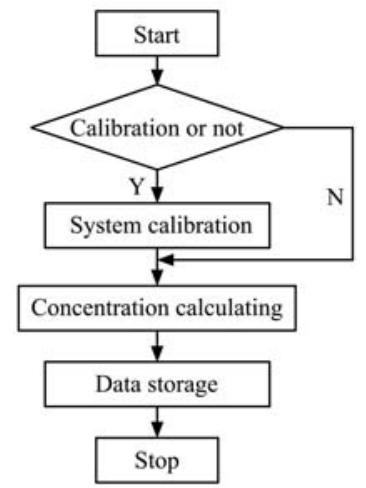

Figure 4 Flowchart of data processing

Formula 1 shows that the relation between gas concentration and sensor resistance is linear. The slope of the curve can be obtained by using two known concentration gases. Equations (2) to (6) are as follows.

$$
\begin{gathered}
\log C=\frac{1}{\alpha}\left(\log A-\log R_{S}\right) \\
\alpha \log C_{1}=\log A-\log R_{S 1} \\
\alpha \log C_{2}=\log A-\log R_{S 2} \\
\alpha=\frac{\log R_{S 2}-\log R_{S 1}}{\log C_{1}-\log C_{2}} \\
\log A=\left(\frac{\log R_{S 2}-\log R_{S 1}}{\log C_{1}-\log C_{2}}\right) \log C_{1}+\log R_{S 1} \\
\log C=\frac{\left(\log C_{1}-\log C_{2}\right)\left(\log R_{S 1}-\log R_{S}\right)}{\log R_{S 2}-\log R_{S 1}}+\log C_{1}
\end{gathered}
$$

Equation (2) shows the calculation for two known gas concentrations and the responding resistance to obtain the concentration of a measured gas.

Sensor resistance is calculated by equation (7).

$$
R_{S}=\frac{V_{C}-R_{L} \times V_{R L}}{V_{R L}}
$$

Where, $R_{S}=$ Sensor resistance $(\mathrm{k} \Omega) ; R_{L}=$ Loading resistor $(\mathrm{k} \Omega)$; $V_{C}=$ Circuit voltage $(\mathrm{kV}) ; V_{R L}=$ Output voltage $(\mathrm{kV})$.

The current of the sensor is calculated by Equation (8).

$$
P_{S}=\frac{V_{C}^{2} \times R_{S}}{\left(R_{S}+R_{L}\right)^{2}}
$$

\subsection{Software development}

VOCs analyzer software was developed based on VB language by adopting modular structure design ideals. Data acquisition, storage, real-time display, concentration calculation and parameter modification were performed by the software. The flowchart of data acquisition and processing is shown in Figure 5.

The characteristics of the measured gas (such as name, content, and concentration) (Figure 6a) can be displayed by the software to help understand the gas properties during data analysis and processing. Also, the data communication port can be chosen and storage time interval can be set. After the basic information is set up, it is possible to measure the concentration of gases by using the VOCs analyzer. The real-time data acquisition interface is shown in Figure 6b, and it can display the current time, the concentration of measured gas and trends in concentration over time.

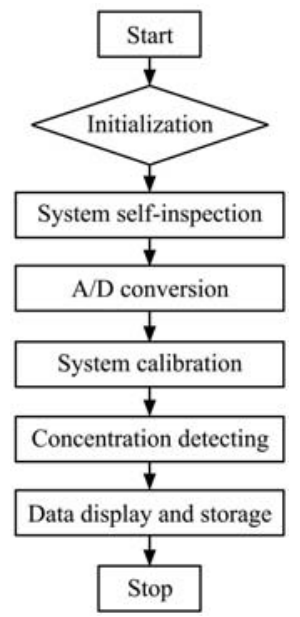

Figure 5 Flowchart of data acquisition and processing
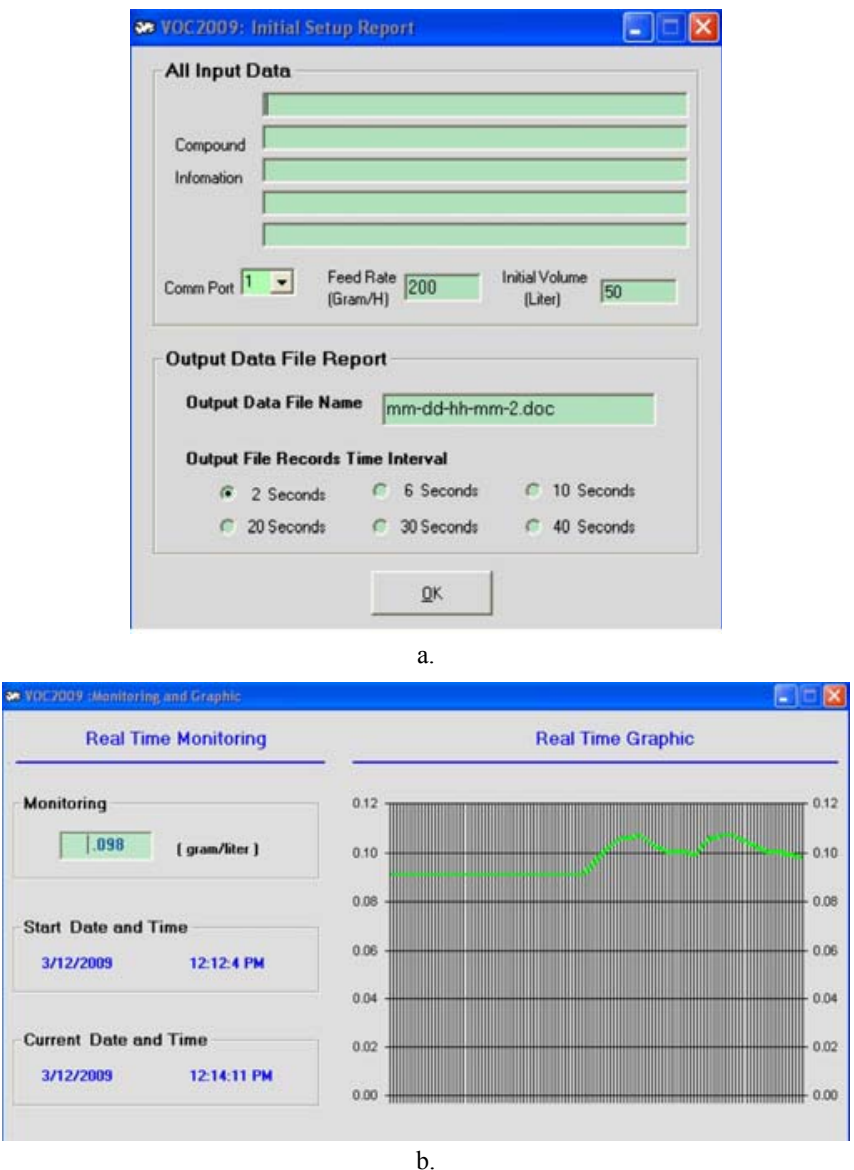

Figure 6 (a) Interface of parameter setting (b) Interface of real-time data acquisition

\section{Results and Discussion}

The new pest control pesticide, Zenivex E20 (Etofenprox, U.S. Zenivex Company) was used in this experiment. Piperonylbutoxide and synergist were not contained in this product, and it can be used directly or diluted. E20 has little impact on the environment and insecticidal effect can be achieved quickly and thoroughly. Its main ingredient is Etofenprox.

\subsection{Test setting}

The VOCs analyzer was put in the field to monitor trend of air concentration after spraying pesticide, and detect the corresponding concentration value (Figure 7a) 
(1) Testing Time: to obtain a relatively constant temperature and air content, the test starts at 6:00am when fields were not subjected to UV irradiation.

(2) Testing Location: the cotton fields in Southern United States Department of Agriculture Research Center; an aircraft spraying pesticide is shown in Figure $7 \mathrm{~b}$.

(3) VOCs analyzer setting: the analyzer was placed in the middle of the field, in height about $1 \mathrm{~m}$.

(4) Concentration: the concentration is $15 \%$ for the first spraying, the second is $10 \%$, and the third is $15 \%$. In order to identify the response of pesticide concentration, two kinds of pesticide are used in the test.

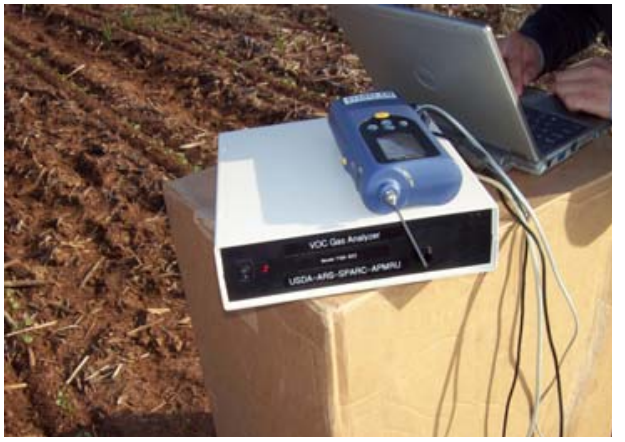

a. VOCs analyzer in the field

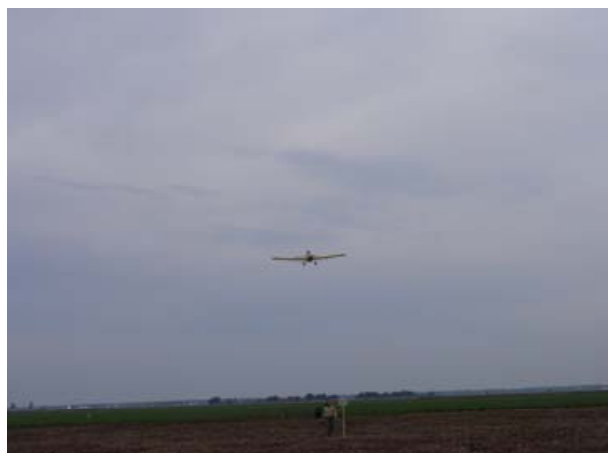

b. Pesticide spraying aircraft

Figure 7 In-field test

\subsection{Testing result analysis}

\subsubsection{Detecting evaluations}

For this test, the pesticide concentration of $15 \%$ was chosen. Due to the effects of wind, the concentration of pesticide is changing continually with pesticides falling to the ground. The changing trend and values are recorded by the VOCs analyzer software, during spraying and for 18 min after (Figure 8).

From the various phases, it can be concluded that the concentration of pesticides were first raised up and then dropped off. Concentration reaches the maximum value of $854.77 \mathrm{ppm}$ at $603 \mathrm{~s}$. According to equation 1, the concentration is $14.22 \%$, with the initial preparation of $15 \%$. The relative error is $5.2 \%$, which is small. Therefore, the VOCs analyzer can be used for detecting the actual pesticide concentration in cotton fields.

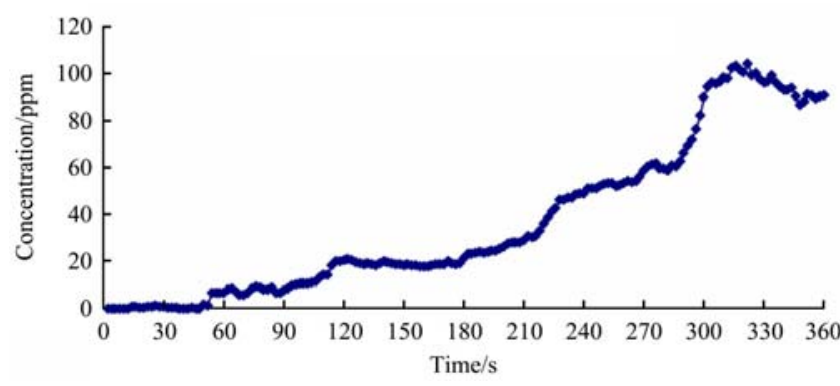

a. Response curve in the initial phase

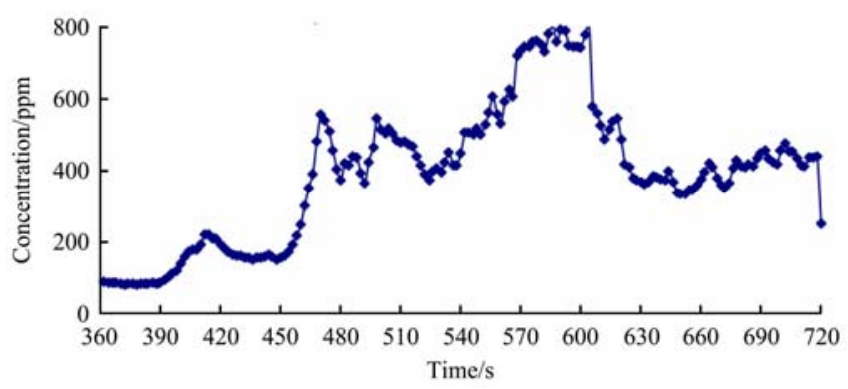

b. Response curve in the middle phase

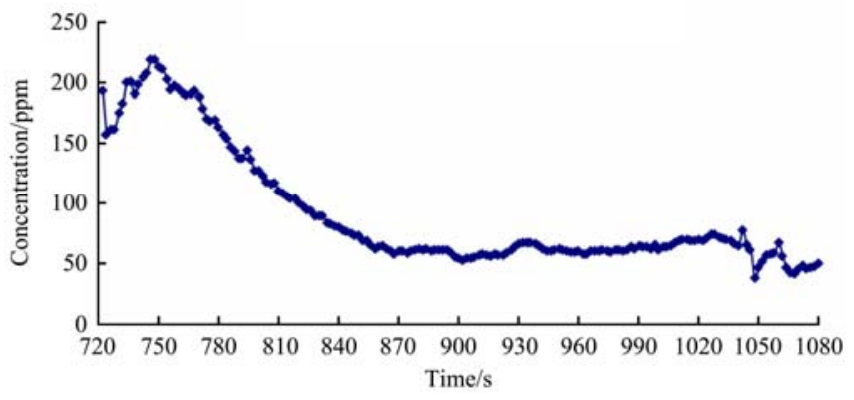

c. Response curve in the later phase

Figure 8 Tendency in the initial phase (0-6 min), middle phase (7-12 $\mathrm{min})$, later phase (8-18 $\mathrm{min})$

\subsubsection{Comparative test}

To identify the concentration-response of VOCs analyzer, two different concentrations of pesticide were prepared. As shown in Figure 9, the concentration-response of $15 \%$ is the light curve, and $10 \%$ is the bold one.

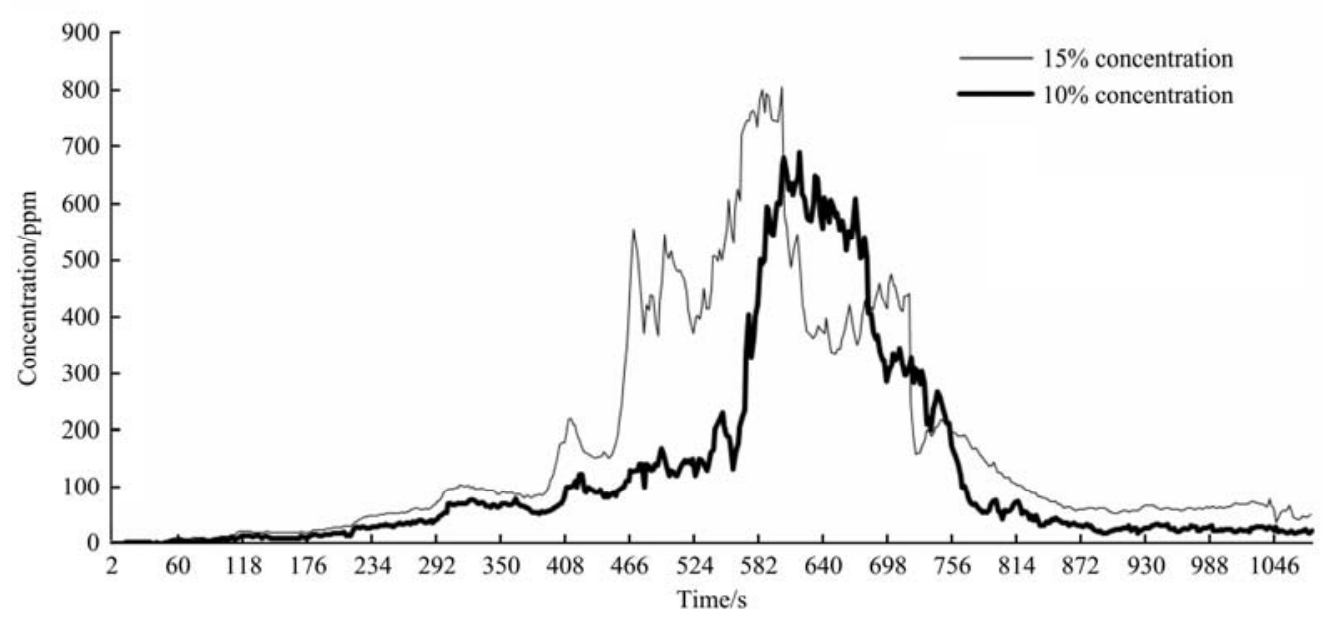

Figure 9 Results of testing 
The maximum value of the two different concentrations can be seen, with the peak of $15 \%$ at $800 \mathrm{ppm}$, and the peak of $10 \%$ at $690 \mathrm{ppm}$. This is due to different testing environments (wind, humidity, temperature). Although the maximum value occurs later and the maximum concentration is not identical to that measured by the sensor, the value is close to the actual concentration of pesticide.

\section{Conclusions}

Based on bionics and artificial olfaction designing ideals, a VOCs analyzer was developed according to the requirements of field-site use. The VOCs analyzer was able to be placed in the field for long-term, consumed a minimal amount of energy, and had high efficiency. It also can be used to accurately and rapidly monitor for odors related to pests and disease. Through field testing, the detection error was revealed to be $5.2 \%$, allows for the conclusion that the VOCs analyzer can be used to detect the actual concentration of pesticide in cotton fields, meeting the design requirements.

\section{[References]}

[1] Constable G A, Bange M P. The yield potential of cotton (Gossypium hirsutum L.). Field Crops Research, 2015; 182: 98-106. doi: 10.1016/ j.fcr.2015.07.017.

[2] Aslam S, Khan S, Ahmed A, et al. The Tale of Cotton Plant: From Wild Type to Domestication, Leading to Its Improvement by Genetic Transformation. American Journal of Molecular Biology, 2020; 10: 91-127. doi: 10.4236/ajmb.2020.102008.

[3] Cauquil J. Cotton-pest control: a review of the introduction of ultra-low-volume (ULV) spraying in sub-Saharan French-speaking Africa. Crop Protection, 1987; 6(1): 38-42. doi: 10.1016/0261-2194(87)90026-3

[4] Japheth D W, Carl A B. Sensitivity of Exserohilum turcicum to demethylation inhibitor fungicides. Crop Protection, 2017; 99: 85-92. doi: 10.1016/j.cropro.2017.05.011.

[5] Semage S N, Pathirana K N, Agampodi S B. Cutaneous leishmaniasis in Mullaitivu, Sri Lanka: a missing endemic district in the leishmaniasis surveillance system. International Journal of Infectious Diseases, 2014; 25: 53-55. doi: 10.1016/j.ijid.2014.03.1382.

[6] Alexandra $\mathrm{M}$, Clarice S, Rafaella $\mathrm{T} P$, et al. Volatile compounds monitoring as indicative of female cattle fertile period using electronic nose. Sensors and Actuators B: Chemical, 2019; 282: 609-616. doi: 10.1016/ j.snb.2018.11.109.

[7] Leonidas M, Evangelos S, Lampros $\mathrm{P}$, et al. Nanoparticle based gas-sensing array for pesticide detection. Journal of Environmental Chemical Engineering, 2018; 6(5): 6641-6646. doi: 10.1016/ j.jece.2018.10.036

[8] Henderson W G, Khaliliana A, Han Y J, et al. Detecting stink bugs/damage in cotton utilizing a portable electronic nose. Computers and Electronics in Agriculture, 2010; 70(1): 157-162. doi: 10.1016/ j.compag.2009.09.019.

[9] Jonsson M. Responses to Oilseed Rape and Cotton Volatiles in Insect Herbivores and Parasitoids. Swedish University of Agricultural Sciences Alnarp, 2005.

[10] Landolt P J. Effects of host plant leaf damage on Cabbage Looper moth attraction and oviposition. Entomologia experimentalis et applicate, 1993 67(11): 79-85. doi: 10.1111/j.1570-7458.1993.tb01654.x.

[11] Dicke M, Beek T A, Posthumus M A, et al. Isolation and identification of volatile kairomone that affects acarine predator-prey interaction, involvement of host plant in its production. Journal of chemical ecology, 1990; 16(2): 381-396. doi: 10.1007/BF01021772.

[12] Liu H, Niu Z Y. Electronic nose technology and its application development. China Measurement \&Test, 2009; 35(5): 6-9.

[13] Li J P. Micro gas sensors and electronic nose study. Chinese Academy of Sciences, 2000.

[14] Li X N. Identification and prevention of common diseases and insect pests of cotton. Modern agricultural science and technology, 2010; 10: 163.

[15] Li Y X. A review on control of important disease and pest insects in cotton crops. agrochemicals research \& application, 2010; 14(2): 4-5.

[16] Mao S C. 60 years review and prospect of global cotton. China cotton circulation economy, 2010; 3: 30-38.

[17] Peng J. Optimized of sensor array and application research. Huazhong University of Science and Technology, 2008.

[18] Yu Y, Wang J, Zhou M. Research developments of electronic nose and its application in processing of agriculture products. Journal of Zhejiang University (Agric. \& Life Sci.), 2003; 29(5): 579-584.

[19] Zhang H M, Wang J, Ye S. Optimized of sensor array and detection of moldy degree for grain by electronic nose. Chinese Journal of Sensors and Actuators, 2007; 6(6): 1207-1210.

[20] Zhu J J. Progress in the development of electronic nose sensor technology. Beijing Biomedical Engineering, 2002; 21(4): 298-302.

[21] Zhu S H, Cao Z L, Yao L L, et al. A new dynamic development of cotton pest insect and disease. Anhui Agri. Sci. Bull, 2006; 12(4): 123-130.

[22] Rains G C, Tomberlin J K, Alessandro M, et al. Limits of volatile chemical detection of a parasitoid wasp, Microplitis croceipes, and an electronic nose: a comparative study. Transactions of the ASAE, 2004; 47(6): 2145-2152. doi: 10.13031/2013.17785. 